\title{
An Interesting Generalization of Fibonacci \& Lucas Sequence
}

\author{
Vandana R. Patel ${ }^{1}$, Devbhadra V. Shah ${ }^{2}$ \\ ${ }^{1}$ Department of Mathematics, Sir P. T. Science College, Modasa, India \\ ${ }^{2}$ Department of Mathematics, Sir P. T. Sarvajanik College of Science, Surat, India
}

\begin{abstract}
In this paper, we consider the generalisation of classical Fibonacci sequence and Lucas sequence. We consider the sequence $\left\{H_{n}\right\}$ defined by the recurrence relation $H_{n}=H_{n-1}+H_{n-2}$; for all $n \geq 2$, with $H_{0}=2 m, H_{1}=k+m$; where $m$, $k$ are fixed integers. The initial conditions are the sum of' $k$ ' times the initial conditions of Fibonacci sequence and ' $m$ ' times the initial conditions of Lucas sequence. Using the technique of generating functions, we obtain the extended Binet formula for $H_{n}$. We obtain some fascinating properties for this sequence. We also establish some amusing identities for this sequence displaying the relation between $H_{n}$, Fibonacci sequence and Lucas sequence.
\end{abstract}

Keywords: Fibonacci sequence; Lucas sequence; generating function; Generalized Fibonacci sequence

\section{Introduction}

Classical Fibonacci sequence can be generalized in many ways: either by preserving the recurrence relation and altering the initial conditions [1], [2], [5], [6]or by conserving the initial conditions of the sequence and modifying the recurrence relation[3], [4]. In this paper, we consider the generalized Fibonacci sequence $\left\{H_{n}\right\}$ defined by the recurrence relation

$\mathrm{H}_{\mathrm{n}}=\mathrm{H}_{\mathrm{n}-1}+\mathrm{H}_{\mathrm{n}-2}$; for all $\mathrm{n} \geq 2$, (1.1)

with $\mathrm{H}_{0}=2 \mathrm{~m}$ and $\mathrm{H}_{1}=\mathrm{k}+\mathrm{m}$; where $\mathrm{m}, \mathrm{k}$ are fixed integers.We obtain extended Binet's formula using the techniques of generating functions and develop the connection formulae as well as negation formula for this sequence.

Here, the initial conditions $\mathrm{H}_{0}$ and $\mathrm{H}_{1}$ are the sum of ' $\mathrm{k}$ ' times the initial conditions of Fibonacci sequence and ' $m$ ' times the initial conditions of Lucas sequencerespectively. First few terms of sequence $\left\{\mathrm{H}_{\mathrm{n}}\right\}$ are: $2 \mathrm{~m}, \mathrm{k}+\mathrm{m}, \mathrm{k}+$ $3 \mathrm{~m}, 2 \mathrm{k}+4 \mathrm{~m}, 3 \mathrm{k}+7 \mathrm{~m}, \ldots$.

\section{Extended Binet's Formula for $\mathbf{H}_{\mathbf{n}}$}

It is well-known that the Binet formula for $\left\{F_{n}\right\}$ and $\left\{L_{n}\right\}$ are given by $F_{n}=\frac{\alpha^{n}-\beta^{n}}{\alpha-\beta}$ and $L_{n}=\alpha^{n}+\beta^{n}$ respectively. Here we obtain the extended Binet's formula for $\left\{\mathrm{H}_{\mathrm{n}}\right\}$.

Lemma 2.1: The generating function for the sequence $\left\{H_{n}\right\}$ is given by

$h(x)=\frac{2 m+(k-m) x}{1-x-x^{2}}$.

Proof: Let $h(x)=\mathrm{H}_{0}+\mathrm{H}_{1} \mathrm{x}+\mathrm{H}_{2} \mathrm{x}^{2}+\cdots$ be the generating function for the sequence $\left\{\mathrm{H}_{\mathrm{n}}\right\}$. Then we get $\mathrm{xh}(\mathrm{x})=\mathrm{H}_{0} \mathrm{x}+\mathrm{H}_{1} \mathrm{x}^{2}+\mathrm{H}_{2} \mathrm{x}^{3}+\cdots+\mathrm{H}_{\mathrm{n}} \mathrm{x}^{\mathrm{n}+1}+\cdots$ and $\mathrm{x}^{2} \mathrm{~h}(\mathrm{x})=\mathrm{H}_{0} \mathrm{x}^{2}+\mathrm{H}_{1} \mathrm{x}^{3}+\mathrm{H}_{2} \mathrm{x}^{4}+\cdots+\mathrm{H}_{\mathrm{n}} \mathrm{x}^{\mathrm{n}+2}+\cdots$

These gives

$$
\begin{array}{rl}
h(x)-x h(x)-x^{2} & h(x) \\
& =H_{0}+\left(-H_{0}+H_{1}\right) x \\
& +\left(-H_{0}-H_{1}+H_{2}\right) x^{2}+\cdots
\end{array}
$$

Using the definition of $\mathrm{H}_{\mathrm{n}}$, we get

$\left(1-\mathrm{x}-\mathrm{x}^{2}\right) \mathrm{h}(\mathrm{x})=2 \mathrm{~m}+(\mathrm{k}-\mathrm{m}) \mathrm{x}$.

$\therefore \mathrm{h}(\mathrm{x})=\sum_{\mathrm{n}=0}^{\infty} \mathrm{H}_{\mathrm{n}} \mathrm{x}^{\mathrm{n}}=\frac{2 \mathrm{~m}+(\mathrm{k}-\mathrm{m}) \mathrm{x}}{1-\mathrm{ax}-\mathrm{bx}^{2}}$, which is the required generating function.

Remark: If we consider $1-x-x^{2}=(1-\alpha x)(1-\beta x)$, then we have

$\alpha+\beta=1, \alpha \beta=-1, \alpha^{3}=2 \alpha+1$. Also we see that $\alpha=\frac{1+\sqrt{5}}{2}$ and $\beta=\frac{1-\sqrt{5}}{2}$.

We now obtain the extended Binet's formula for the sequence $\left\{\mathrm{H}_{\mathrm{n}}\right\}$.

Theorem 2.2: $\mathrm{H}_{\mathrm{n}}=\mathrm{k}\left(\frac{\alpha^{\mathrm{n}}-\beta^{\mathrm{n}}}{\alpha-\beta}\right)+\mathrm{m}\left(\alpha^{\mathrm{n}}+\beta^{\mathrm{n}}\right)$.

Proof: If we consider $h(x)=\frac{2 m+(k-m) x}{1-x-x^{2}}=\frac{A}{1-\alpha x}+\frac{B}{1-\beta x}$, then it can be seen that

$\mathrm{A}=\frac{2 \mathrm{~m} \alpha+(\mathrm{k}-\mathrm{m})}{\alpha-\beta}$ and $\mathrm{B}=-\frac{2 \mathrm{~m} \beta+(\mathrm{k}-\mathrm{m})}{\alpha-\beta}$.

Thus $\mathrm{h}(\mathrm{x})=\frac{2 \mathrm{~m}+(\mathrm{k}-\mathrm{m}) \mathrm{x}}{1-\mathrm{x}-\mathrm{x}^{2}}=\frac{\frac{2 \mathrm{~m} \alpha+(\mathrm{k}-\mathrm{m})}{\alpha-\beta}}{1-\alpha \mathrm{x}}+-\frac{\left(\frac{2 \mathrm{~m} \beta+(\mathrm{k}-\mathrm{m})}{\alpha-\beta}\right)}{1-\beta \mathrm{x}}$

$=\frac{2 \mathrm{~m} \alpha+(\mathrm{k}-\mathrm{m})}{\alpha-\beta} \sum_{\mathrm{n}=0}^{\infty} \alpha^{\mathrm{n}} \mathrm{x}^{\mathrm{n}}-\left(\frac{2 \mathrm{~m} \beta+(\mathrm{k}-\mathrm{m})}{\alpha-\beta}\right) \sum_{\mathrm{n}=0}^{\infty} \beta^{\mathrm{n}} \mathrm{x}^{\mathrm{n}}$

$=2 \mathrm{~m} \sum_{\mathrm{n}=0}^{\infty}\left(\frac{\alpha^{\mathrm{n}+1}-\beta^{\mathrm{n}+1}}{\alpha-\beta}\right) \mathrm{x}^{\mathrm{n}}+(\mathrm{k}-\mathrm{m}) \sum_{\mathrm{n}=0}^{\infty}\left(\frac{\alpha^{\mathrm{n}}-\beta^{\mathrm{n}}}{\alpha-\beta}\right) \mathrm{x}^{\mathrm{n}}$.

This gives

$\sum_{\mathrm{n}=0}^{\infty} \mathrm{H}_{\mathrm{n}} \mathrm{x}^{\mathrm{n}}=\left[2 \mathrm{~m} \sum_{\mathrm{n}=0}^{\infty}\left(\frac{\alpha^{\mathrm{n}+1}-\beta^{\mathrm{n}+1}}{\alpha-\beta}\right)+(\mathrm{k}-\right.$

m) $n=0 \infty \alpha n-\beta n \alpha-\beta x n$.

Thus

$$
\begin{aligned}
\mathrm{H}_{\mathrm{n}}= & 2 \mathrm{~m}\left(\frac{\alpha^{\mathrm{n}+1}-\beta^{\mathrm{n}+1}}{\alpha-\beta}\right)+(\mathrm{k}-\mathrm{m})\left(\frac{\alpha^{\mathrm{n}}-\beta^{\mathrm{n}}}{\alpha-\beta}\right) \\
= & \mathrm{k}\left(\frac{\alpha^{\mathrm{n}}-\beta^{\mathrm{n}}}{\alpha-\beta}\right)+\mathrm{m}\left(\frac{\alpha^{\mathrm{n}+1}-\beta^{\mathrm{n}+1}}{\alpha-\beta}\right)+ \\
& \frac{\mathrm{m}}{\alpha-\beta}\left(\alpha^{\mathrm{n}+1}-\beta^{\mathrm{n}+1}-\alpha^{\mathrm{n}}+\beta^{\mathrm{n}}\right) \\
= & \mathrm{k}\left(\frac{\alpha^{\mathrm{n}}-\beta^{\mathrm{n}}}{\alpha-\beta}\right)+\mathrm{m}\left(\frac{\alpha^{\mathrm{n}+1}-\beta^{\mathrm{n}+1}}{\alpha-\beta}\right)+ \\
& \frac{\mathrm{m}}{\alpha-\beta}\left(-\frac{\alpha^{\mathrm{n}}}{\beta}+\frac{\beta^{\mathrm{n}}}{\alpha}+\frac{\alpha^{\mathrm{n}-1}}{\beta}-\frac{\beta^{\mathrm{n}-1}}{\alpha}\right)
\end{aligned}
$$




\section{International Journal of Science and Research (IJSR) \\ ISSN (Online): 2319-7064}

Index Copernicus Value (2013): 6.14 | Impact Factor (2014): 5.611

$$
\begin{gathered}
=\mathrm{k}\left(\frac{\alpha^{\mathrm{n}}-\beta^{\mathrm{n}}}{\alpha-\beta}\right)+\mathrm{m}\left(\frac{\alpha^{\mathrm{n}+1}-\beta^{\mathrm{n}+1}}{\alpha-\beta}\right)+ \\
\frac{\mathrm{m}}{\alpha-\beta}\left(\frac{\alpha^{\mathrm{n}-1}}{\beta}(1-\alpha)-\frac{\beta^{\mathrm{n}-1}}{\alpha}(1-\beta)\right) \\
=\mathrm{k}\left(\frac{\alpha^{\mathrm{n}}-\beta^{\mathrm{n}}}{\alpha-\beta}\right)+\mathrm{m}\left(\frac{\alpha^{\mathrm{n}+1}-\beta^{\mathrm{n}+1}}{\alpha-\beta}\right) \\
\quad+\frac{\mathrm{m}}{\alpha-\beta}\left(\frac{\alpha^{\mathrm{n}-1}}{\beta} \beta-\frac{\beta^{\mathrm{n}-1}}{\alpha} \alpha\right) \\
=\mathrm{k}\left(\frac{\alpha^{\mathrm{n}}-\beta^{\mathrm{n}}}{\alpha-\beta}\right)+\frac{\mathrm{m}}{\alpha-\beta}\left(\alpha^{\mathrm{n}+1}-\beta^{\mathrm{n}+1}+\alpha^{\mathrm{n}-1}-\beta^{\mathrm{n}-1}\right) \\
=\mathrm{k}\left(\frac{\alpha^{\mathrm{n}}-\beta^{\mathrm{n}}}{\alpha-\beta}\right)+\frac{\mathrm{m}}{\alpha-\beta}\left(\alpha^{\mathrm{n}}\left(\alpha+\frac{1}{\alpha}\right)-\beta^{\mathrm{n}}\left(\beta+\frac{1}{\beta}\right)\right) \\
=k\left(\frac{\alpha^{n}-\beta^{n}}{\alpha-\beta}\right)+\frac{m}{\alpha-\beta}\left(\alpha^{n}(\alpha-\beta)-\beta^{n}(\beta-\alpha)\right) \\
=k\left(\frac{\alpha^{n}-\beta^{n}}{\alpha-\beta}\right)+\frac{m}{\alpha-\beta}\left(\left(\alpha^{n}+\beta^{n}\right)(\alpha-\beta)\right) \\
=k\left(\frac{\alpha^{n}-\beta^{n}}{\alpha-\beta}\right)+m\left(\alpha^{n}+\beta^{n}\right), \text { as required. }
\end{gathered}
$$

We use this result to express $H_{n}$ as a linear combination of $F_{n}$ and $L_{n}$.

Corollary 2.3: $H_{n}=k F_{n}+m L_{n}$.

Proof: Using the Binet's formula for the sequences $\left\{H_{n}\right\}$, Fibonacci Sequence $\left\{F_{n}\right\}$ and Lucas Sequence $\left\{L_{n}\right\}$, we get

$$
\begin{aligned}
& H_{n}=k\left(\frac{\alpha^{n}-\beta^{n}}{\alpha-\beta}\right)+m\left(\alpha^{n}+\beta^{n}\right) \\
& =k F_{n}+m L_{n} .
\end{aligned}
$$

\section{Some Summation Formulae for $\mathbf{H}_{n}$}

We first find the value of sum of first $\mathrm{n}$ terms of the sequence $\left\{H_{n}\right\}$.

Lemma 3.1: $H_{1}+H_{2}+\cdots+H_{n}=H_{n+2}-(3 m+k)$.

Proof:

We have $H_{n}=H_{n-1}+H_{n-2} \Rightarrow H_{n-2}=H_{n}-H_{n-1}$.

Substituting $n=3,4,5, \ldots$ successively, we get

$H_{1}=H_{3}-H_{2}, H_{2}=H_{4}-H_{3}, \ldots, H_{n}=H_{n+2}-H_{n+1}$.

Adding all these equations, we get

$H_{1}+H_{2}+\cdots+H_{n}=\left(H_{3}+H_{4}+\cdots+H_{n+2}\right)-$

$\left(H_{2}+H_{3}+\cdots+H_{n+1}\right)$.

$\therefore \sum_{i=1}^{n} H_{i}=H_{n+2}-H_{2}=H_{n+2}-(3 m+k)$, as required.

Lemma 3.2: $H_{1}+H_{3}+\cdots+H_{2 n-1}=H_{2 n}-2 m$.

Proof: We have $H_{n-1}=H_{n}-H_{n-2}$. Replacing $\mathrm{n}$ by $2,4,6, \ldots$ successively, we get

$H_{1}=H_{2}-H_{0}, H_{3}=H_{4}-H_{2}, \ldots, H_{2 n-1}=H_{2 n}-H_{2 n-2}$.

Adding all these equations, we get

$H_{1}+H_{3}+\cdots+H_{2 n-1}=\left(H_{2}+H_{4}+\cdots+H_{2 n}\right)-$

$\left(H_{0}+H_{2}+\cdots+H_{2 n-2}\right)$.

$\therefore \sum_{i=1}^{n} H_{2 i-1}=H_{2 n}-H_{0}=H_{2 n}-2 m$.
Lemma 3.3: $H_{2}+H_{4}+\cdots+H_{2 n}=H_{2 n+1}-(k+m)$.

This result can be proved easily using the earlier techniques or otherwise.

Lemma 3.4: $H_{1}-H_{2}+H_{3}-H_{4}+\cdots+(-1)^{n+1} H_{n}=$ $(-1)^{n+1} H_{n-1}+k-m$.

Proof: From Lemma 3.2 and 3.3, we get

$$
\begin{aligned}
& H_{1}-H_{2}+H_{3}-H_{4}+\cdots+H_{2 n-1}-H_{2 n} \\
= & =H_{2 n}-2 m-H_{2 n+1}+(k+m) \\
= & -H_{2 n-1}+k-m .
\end{aligned}
$$

Adding $H_{2 n+1}$ on both sides we get,

$$
\begin{aligned}
& H_{1}-H_{2}+H_{3}-H_{4}+\cdots+H_{2 n-1}-H_{2 n}+H_{2 n+1} \\
= & H_{2 n}+k-m .
\end{aligned}
$$

Combining last two equations, we write

$H_{1}-H_{2}+H_{3}-H_{4}+\cdots+(-1)^{n+1} H_{n}=$ $(-1)^{n+1} H_{n-1}+k-m$.

We next find the expression for the sum of squares of first $\mathrm{n}$ terms of sequence $\left\{H_{n}\right\}$.

Lemma 3.5: $\quad H_{1}^{2}+H_{2}^{2}+H_{3}^{2}+\cdots+H_{n}^{2}=H_{n} H_{n+1}-$ $2 m(k+m)$.

Proof :We have $H_{n} H_{n+1}=H_{n}\left(H_{n}+H_{n-1}\right)$

$$
\begin{aligned}
& =H_{n}^{2}+H_{n} H_{n-1} \\
& =H_{n}^{2}+\left(H_{n-1}+H_{n-2}\right) H_{n-1} \\
& =H_{n}^{2}+H_{n-1}^{2}+H_{n-2} H_{n-1}
\end{aligned}
$$

Continuing in this way, we get

$$
H_{n} H_{n+1}=H_{n}^{2}+H_{n-1}^{2}+\cdots+H_{1}^{2}+H_{1} H_{0}
$$

$\therefore H_{n} H_{n+1}=H_{n}^{2}+H_{n-1}^{2}+\cdots+H_{1}^{2}+2 m(k+m)$.Thus, $H_{1}^{2}+H_{2}^{2}+H_{3}^{2}+\cdots+H_{n}^{2}=H_{n} H_{n+1}-2 m(k+m)$.

Corollary 3.6: $H_{n}^{2}=H_{n} H_{n+1}-H_{n-1} H_{n}$; for $n \geq 1$.

Proof: From the proof of Lemma 3.5, we have $H_{n} H_{n+1}=$ $H_{n}^{2}+H_{n-1}^{2}+\cdots+H_{1}^{2}+H_{1} H_{0}$ and $H_{n-1} H_{n}=H_{n-1}^{2}+\cdots+$ $H_{1}^{2}+H_{1} H_{0}$.

Subtracting we get $H_{n}^{2}=H_{n} H_{n+1}-H_{n-1} H_{n} ; n \geq 1$.

Lemma 3.7: $H_{n}^{2}+H_{n+1}^{2}=H_{1} H_{2 n+1}+H_{0} H_{2 n}$.

Proof: We prove the result by induction on $\mathrm{n}$.

For $n=1, H_{1}^{2}+H_{2}^{2}=(k+m)^{2}+(k+3 m)^{2}=2 k^{2}+$ $10 \mathrm{~m}^{2}+8 \mathrm{~km}$.

Also $\quad H_{1} H_{3}+H_{0} H_{2}=(k+m)(2 k+4 m)+2 m(k+$ $3 m=2 k 2+10 m 2+8 k m$.

Thus result is true for $n=1$. Assume that result is true for all integers $n$ not exceeding some positive integer $k$. Thus $H_{k}^{2}+H_{k+1}^{2}=H_{1} H_{2 k+1}+H_{0} H_{2 k}$.Now,

$H_{k+1}^{2}+H_{k+2}^{2}=H_{k+1} H_{k+1}+H_{k+2} H_{k+2}$

$=H_{k+1}\left(H_{1} F_{k+1}+F_{k} H_{0}\right)+H_{k+2}\left(H_{1} F_{k+2}+F_{k+1} H_{0}\right)$

$=H_{1}\left(H_{k+1} F_{k+1}+F_{k+2} H_{k+2}\right)+H_{0}\left(H_{k+1} F_{k}+F_{k+1} H_{k+2}\right)$

$=H_{1} H_{2 k+3}+H_{0} H_{2 k+2}$.

Thus result is true for $n=k+1$ also. This proves the result.

We use extended Binet formula for $H_{n}$ to prove the following result. 


\section{International Journal of Science and Research (IJSR) \\ ISSN (Online): 2319-7064}

Index Copernicus Value (2013): 6.14 | Impact Factor (2014): 5.611

Lemma 3.8: $H_{3}+H_{6}+H_{9}+\cdots+H_{3 n}=\frac{1}{2}\left(H_{3 n+2}-(k+\right.$ $3 m)$.

Proof: By using extended Binet's formula from Theorem 2.2 we get

$$
\begin{aligned}
& H_{3}+H_{6}+H_{9}+\cdots+H_{3 n}=k\left(\frac{\alpha^{3}-\beta^{3}}{\sqrt{5}}\right)+m\left(\alpha^{3}+\beta^{3}\right)+ \\
& k\left(\frac{\alpha^{6}-\beta^{6}}{\sqrt{5}}\right)+m\left(\alpha^{6}+\beta^{6}\right)+\cdots \\
& +k\left(\frac{\alpha^{3 n}-\beta^{3 n}}{\sqrt{5}}\right)+m\left(\alpha^{3 n}+\beta^{3 n}\right) \\
& =\frac{k}{\sqrt{5}}\left[\left(\alpha^{3}+\alpha^{6}+\cdots+\alpha^{3 n}\right)-\left(\beta^{3}+\beta^{6}+\cdots+\beta^{3 n}\right)\right] \\
& +m\left[\left(\alpha^{3}+\alpha^{6}+\cdots+\alpha^{3 n}\right)-\left(\beta^{3}+\beta^{6}+\cdots+\beta^{3 n}\right)\right] \\
& =\frac{k}{\sqrt{5}}\left[\left(\frac{\alpha^{3 n+3}-\alpha^{3}}{\alpha^{3}-1}\right)-\left(\frac{\beta^{3 n+3}+\beta^{3}}{\beta^{3}-1}\right)\right] \\
& +m\left[\left(\frac{\alpha^{3 n+3}-\alpha^{3}}{\alpha^{3}-1}\right)-\left(\frac{\beta^{3 n+3}+\beta^{3}}{\beta^{3}-1}\right)\right] \\
& =\frac{k}{\sqrt{5}}\left[\left(\frac{\alpha^{3 n+2}-\alpha^{2}}{2}\right)-\left(\frac{\beta^{3 n+2}+\beta^{2}}{2}\right)\right] \\
& +m\left[\left(\frac{\alpha^{3 n+2}-\alpha^{2}}{2}\right)+\left(\frac{\beta^{3 n+2}+\beta^{2}}{2}\right)\right] \\
& =\frac{1}{2}\left[\begin{array}{c}
\left(k\left(\frac{\alpha^{3 n+2}-\beta^{3 n+2}}{\sqrt{5}}\right)+m\left(\alpha^{3 n+2}+\beta^{3 n+2}\right)\right) \\
-\left(k\left(\frac{\alpha^{3 n+2}-\beta^{3 n+2}}{\sqrt{5}}\right)+m\left(\alpha^{3 n+2}+\beta^{3 n+2}\right)\right)
\end{array}\right] \\
& =\frac{1}{2}\left[H_{3 n+2}-H_{2}\right]
\end{aligned}
$$

Thus $\sum_{i=1}^{n} H_{3 i}=\frac{1}{2}\left[H_{3 n+2}-(k+3 m)\right]$, as required.

$$
\begin{aligned}
& \text { Lemma 3.9: } \quad H_{5}+H_{8}+H_{11}+\cdots+H_{3 n+2}= \\
& \frac{1}{2}\left(H_{3 n+4}-(3 k+7 m)\right) \text {. }
\end{aligned}
$$

This result can be proved easily by the techniques used above.

We now derive the extended Cassini's identity which connects three consecutive $H_{n}$ 's together.

Lemma 3.10: $H_{n+1} H_{n-1}-H_{n}^{2}=(-1)^{n+1}\left(5 m^{2}-k^{2}\right)$.

Proof: Here also we use the technique of mathematical induction over $\mathrm{n}$.

It is easy to see that result is true for $n=1$, for

$H_{2} H_{0}-H_{1}^{2}=(k+3 m) 2 m-(k+m)^{2}=$

$(-1)^{2}\left(5 m^{2}-k^{2}\right)$.

Now suppose that it is true for all positive integers not exceeding a positive integer ' $\mathrm{t}$ '. Thus $H_{t+1} H_{t-1}-H_{t}^{2}=$ $(-1)^{t+1}\left(5 m^{2}-k^{2}\right)$ holds.

Now adding and subtracting $H_{t} H_{t+1}$ onleft side we get,

$$
\begin{gathered}
H_{t+1} H_{t-1}-H_{t}^{2}+H_{t} H_{t+1}-H_{t} H_{t+1} \\
=(-1)^{t+1}\left(5 m^{2}-k^{2}\right) \\
\therefore H_{t+1}\left(H_{t}+H_{t-1}\right)-H_{t}^{2}-H_{t} H_{t+1} \\
=(-1)^{t+1}\left(5 m^{2}-k^{2}\right) \\
\therefore H_{t+1}^{2}-H_{t}\left(H_{t}+H_{t+1}\right)=(-1)^{t+1}\left(5 m^{2}-k^{2}\right) \\
\therefore-\left(H_{t} H_{t+2}-H_{t+1}^{2}\right)=(-1)^{t+1}\left(5 m^{2}-k^{2}\right)
\end{gathered}
$$

$\therefore H_{t} H_{t+2}-H_{t+1}^{2}=(-1)^{t+2}\left(5 m^{2}-k^{2}\right)$, which is precisely our identity when $n=t+1$. This proves the result by the induction.

\section{Connection Formulae}

Here we establish some amusing identities for the sequence $\left\{H_{n}\right\}$ displaying its relation with Fibonacci sequence and Lucas sequence.

\section{Theorem 4.1:}

(i) $H_{n+1}+H_{n-1}=(k+m) L_{n}+2 m L_{n-1}$ $H_{n-1}=(k+m) F_{n}+2 m F_{n-1}$;

where $n \geq 1$

Proof: We again use the induction technique to prove first result. Likewise second result can also be proved by induction.

For $n=1$ we have $H_{2}+H_{0}=k+5 m$. Also $(k+$ $m L 1+2 m \angle 0=k+5 m$. Thus result is true for $n=1$.

We assume that the result is true for all integers not exceeding any positive integer ' $\mathrm{t}$ '. Thus $H_{t+1}+H_{t-1}=$ $(k+m) L_{t}+2 m L_{t-1}$ holds. Now,

$$
\begin{gathered}
H_{t+2}+H_{t}=H_{t+1}+H_{t}+H_{t-1}+H_{t-2} \\
=\left(H_{t+1}+H_{t-1}\right)+\left(H_{t}+H_{t-2}\right) \\
=(k+m) L_{t}+2 m L_{t-1}+(k+m) L_{t-1}+2 m L_{t-2} \\
=(k+m)\left(L_{t}+L_{t-1}\right)+2 m\left(L_{t-1}+L_{t-2}\right) \\
=(k+m) L_{t+1}+2 m L_{t} .
\end{gathered}
$$

This proves the result for $n=t+1$ and eventually for all positive integers $n$.

We next prove more generalized form of extended Catalan's identity which connects three consecutive $H_{n}$ 's with suffixes in arithmetic progression with $F_{n}$, for fixed n.

Theorem 4. $H_{n}^{2}-H_{n+r} H_{n-r}=(-1)^{n-r-1}\left(5 m^{2}-\right.$ $\left.k^{2}\right) F_{r}^{2}$.

Proof: Using theorem 2.2, we have

$$
\begin{gathered}
H_{n}^{2}-H_{n+r} H_{n-r}=\left[k\left(\frac{\alpha^{n}-\beta^{n}}{\sqrt{5}}\right)+m\left(\alpha^{n}+\beta^{n}\right)\right]^{2} \\
-\left[k\left(\frac{\alpha^{n+r}-\beta^{n+r}}{\sqrt{5}}\right)\right. \\
\left.+m\left(\alpha^{n+r}+\beta^{n+r}\right)\right] \\
\times\left[k\left(\frac{\alpha^{n-r}-\beta^{n-r}}{\sqrt{5}}\right)+m\left(\alpha^{n-r}+\beta^{n-r}\right)\right] \\
=\frac{k^{2}}{5}\left(-2 \alpha^{n} \beta^{n}+\alpha^{n} \beta^{n}\left(\frac{\beta}{\alpha}\right)^{r}+\alpha^{n} \beta^{n}\left(\frac{\alpha}{\beta}\right)^{r}\right) \\
+m^{2}\left(\begin{array}{c}
\left.2 \alpha^{n} \beta^{n}-\alpha^{n} \beta^{n}\left(\frac{\beta}{\alpha}\right)^{r}\right) \\
-\alpha^{n} \beta^{n}\left(\frac{\alpha}{\beta}\right)^{r}
\end{array}\right) \\
=\frac{k^{2}(\alpha \beta)^{n}}{5}\left[\frac{\alpha^{2 r}+\beta^{2 r}-2 \alpha^{r} \beta^{r}}{(\alpha \beta)^{r}}\right] \\
+m^{2}(\alpha \beta)^{n}\left[\frac{\alpha^{2 r}+\beta^{2 r}-2 \alpha^{r} \beta^{r}}{(\alpha \beta)^{r}}\right] \\
=(\alpha \beta)^{n-r}\left(\alpha^{r}-\beta^{r}\right)^{2}\left[\frac{k^{2}}{5}-m^{2}\right]
\end{gathered}
$$




\section{International Journal of Science and Research (IJSR)

$$
\begin{gathered}
=(-1)^{n-r} \frac{\left(\alpha^{r}-\beta^{r}\right)^{2}}{5}\left(k^{2}-5 m^{2}\right) \\
=(-1)^{n-r-1}\left(5 m^{2}-k^{2}\right) F_{r}^{2} .
\end{gathered}
$$

We finally derive an interesting result which connects $H_{-n}$ with Fibonacci sequence and Lucas sequence.

Lemma 4.3: $H_{-n}=(-1)^{n}\left(m L_{n}-k F_{n}\right)$.

Proof: We have $H_{n}=k\left(\frac{\alpha^{n}-\beta^{n}}{\sqrt{5}}\right)+m\left(\alpha^{n}+\beta^{n}\right)$

$$
\begin{array}{r}
\therefore H_{-n}=k\left(\frac{\alpha^{-n}-\beta^{-n}}{\sqrt{5}}\right)+m\left(\alpha^{-n}+\beta^{-n}\right) \\
=\frac{k}{\sqrt{5}}\left(\frac{\beta^{n}-\alpha^{n}}{(\alpha \beta)^{n}}\right)+m\left(\frac{\beta^{n}+\alpha^{n}}{(\alpha \beta)^{n}}\right) \\
=\frac{k}{\sqrt{5}}(-1)^{n+1}\left(\alpha^{n}-\beta^{n}\right)+m(-1)^{n}\left(\alpha^{n}+\beta^{n}\right) .
\end{array}
$$

Hence, $H_{-n}=(-1)^{n}\left(m L_{n}-k F_{n}\right)$.

\section{References}

[1] Badshah, V. H., Teeth, M. S., \& Dar, M. M. (2012). Generalized Fibonacci-Like Sequence and its Properties. Int. J. Contemp. Math. Sciences, 7(24), $1155-1164$.

[2] Bilgici, G. (2014). New Generalizations of Fibonacci and Lucas Sequences, 8(29), 1429-1437.

[3] Catarino, P. (2014). On Some Identities for k Fibonacci Sequence, 9(1), 37-42.

[4] Horadam, A. F. (1965). Basic properties of a certain generalized sequence of numbers. The Fibonacci Quarterly, 2, 161-176.

[5] Sanjay Harne, Bijendra Singh, S. P. (2014). Generalized Fibonacci-Like Sequence and Fibonacci Sequence. Int. J. Contemp. Math. Sciences, 9(5), 235241.

[6] Singh, B., Sikhwal, O., \& Bhatnagar, S. (2010). Fibonacci-Like Sequence andits Properties.Int. J. Contemp. Math. Sciences, 5(18), 859-868 